\title{
Video Streaming over IP using the DASH Technique
}

\author{
Christian Hoppe ${ }^{1}$ and Tadeus $\mathrm{Uhl}^{2}$ \\ ${ }^{1}$ Nextragen Solutions GmbH Flensburg, Handewitt, Germany \\ ${ }^{2}$ Maritime University of Szczecin, Szczecin, Poland
}

https://doi.org/10.26636/jtit.2018.125318

\begin{abstract}
Modern Internet serves as a high-performance platform for an ever-increasing number of services. Some of them - video services in particular - are of the broadband variety. So, it is not surprising that segments of networks can rapidly become congested, which may lead to a deterioration in quality. It is hoped that the new MPEG-DASH technique will alleviate congestion. This paper contains an analysis of the new technology's impact on the quality of service in IP networks. It also explains a new numerical tool QoSCalc(DASH) that has been used to analyze the DASH method in different scenarios. The results are presented graphically and interpreted.
\end{abstract}

Keywords-H.265, IP network technology, MPEG-DASH, QoS measurement techniques, QoS measurement environment, video streaming, VP9.

\section{Introduction}

There is currently no better communication technology available than IP. It is extremely flexible and has been implemented worldwide. The modern Internet of Things (IoT) concept is also based on IP technology. The number of applications is growing rapidly, with video applications especially on the increase recently. It is common knowledge that video services are extremely resource consuming. So, congestion will occur rapidly in certain segments of the Internet. Packet losses, end-to-end delays and jitter are the inevitable consequences. They will be reflected by a rapid deterioration in service quality, and customer satisfaction will plummet. New techniques will have to be adopted to address the situation. One such new technique is the MPEG-DASH method (Dynamic Adaptive Streaming over HTTP), that was standardized in 2012 as ISO/IEC 23009-1:2012 [1]. What effects does it have on QoS in IP networks? The work described in this paper aimed to find an answer to that question.

In general, two models are used to determine the quality of service/quality of experience (QoS/QoE) in a network: the dual-ended model and the single-ended model [2]. In the case of the dual-ended model, two signals are used: the original signal and the degraded signal. These two signals are available uncompressed. For this reason, measurements can be carried out for both QoE (a subjective evaluation) and Quality of Service (an objective evaluation). In the case of the single-ended model, only the impaired signal (compressed) is available. This allows only an objective evaluation of QoS to be made.

The most widely used QoE measurement techniques for video services over IP (VSoIP) are currently PEVQ (J.247) [3] and VQuad-HD (J.341) [4]. These techniques are very accurate. They are, however, time-consuming and can often only be implemented with a license. Both algorithms incorporate an emulation of the human eye, and so, one can justifiably speak of QoE values here. It is also the reason why these $\mathrm{QoE}$ methods were chosen for the analyses described in this paper.

This paper begins with a brief explanation of the MPEGDASH technique. It then goes on to introduce a new numerical tool to analyze the DASH method. The analysis scenarios are then described. The results obtained are presented graphically and interpreted. The paper concludes with a summary and an outlook on future work.

\section{How Does MPEG-DASH Work?}

Dynamic Adaptive Streaming over HTTP (DASH), also known as MPEG-DASH, is an adaptive bitrate streaming technique that enables high-quality streaming of media content over the Internet hosted by conventional HTTP web servers. Like Apple's HTTP Live Streaming (HLS) solution, MPEG-DASH works by breaking the content into a sequence of small HTTP-based file segments, each segment containing a short interval of playback time of content that is potentially many hours in duration, such as a movie or a live broadcast of a sports event. The content is made available at a variety of different bitrates, i.e. alternative segments encoded at different bitrates covering aligned short intervals of playback time. While the content is being played back by an MPEG-DASH client, the client automatically selects, from the alternatives, the next segment to download and play based on current network conditions. The client selects the segment with the highest bitrate possible that can be downloaded in time for playback without causing stalls or re-buffering events in the playback. Thus, an MPEG-DASH client can seamlessly adapt to changing network conditions and can provide high-quality playback with fewer stalls or re-buffering events. 
MPEG-DASH is the first adaptive bitrate HTTP-based streaming solution to become an international standard. MPEG-DASH should not be confused with a transport protocol - the transport protocol that MPEG-DASH uses is TCP. MPEG-DASH uses existing HTTP web server infrastructure that is used for delivery of essentially all online content. It allows devices such as Internet-connected TVs, set-top boxes, personal computers, smartphones, tablets, etc., to consume multimedia content (video, TV, radio, etc.) delivered via the Internet, coping with variable Internet receiving conditions. Standardizing an adaptive streaming solution was intended to promote confidence on the market that the solution can be adopted for universal deployment, compared to similar but proprietary solutions such as Microsoft Smooth Streaming, or Adobe HDS. DASH is codec-agnostic, which means it can use content encoded with any coding format, e.g. H.264, H.265, VP9 [5].

\section{A New Tool QoSCalc(DASH)}

The QoSCalc(IPTV) tool described in a previous paper [6] can take a reference video and encode it with a specified codec and different parameters. Afterwards, it can apply impairments in the form of packet loss and compare the resulting video with the reference. This tool serves as a basis for analyzing MPEG-DASH. However, MPEG-DASH introduces the need to change the workflow of the measurements as more than one format to encode is used.

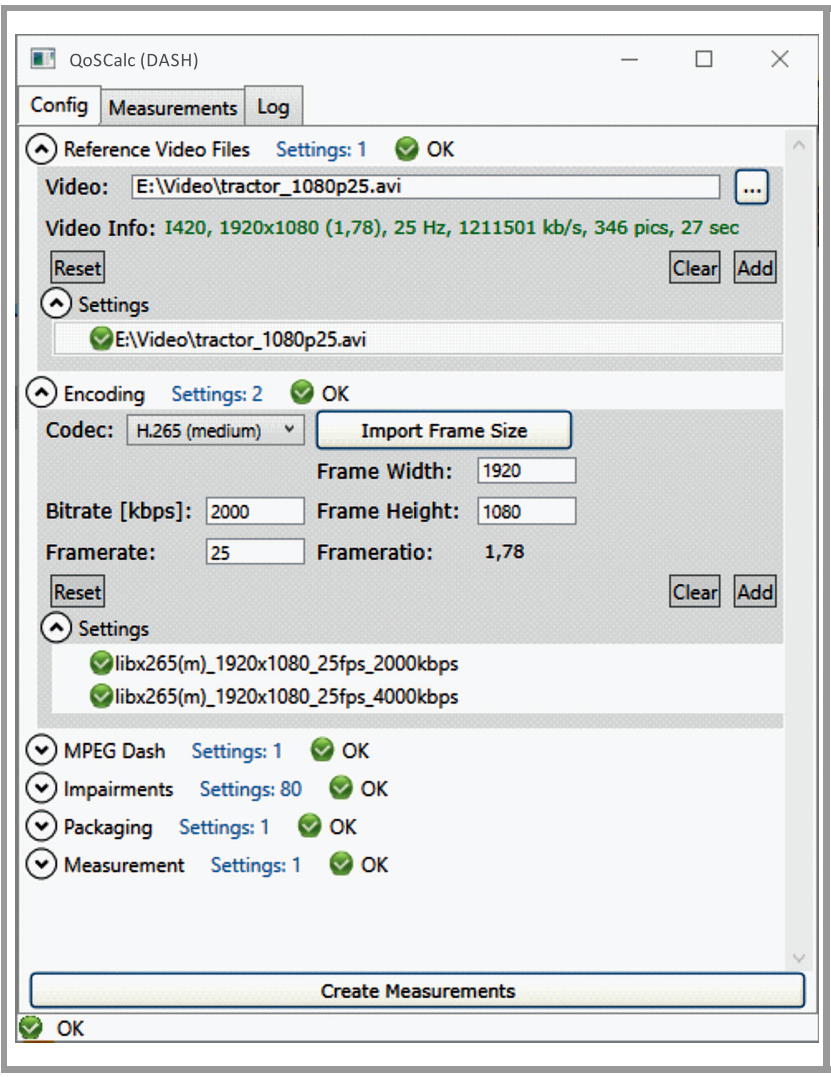

Fig. 1. Reference video and encoding settings for the QoSCalc(DASH) tool.
The most significant changes to the tool QoSCalc(DASH) is the need to encode the reference video in different formats and to make sure that such encoded videos can be seamlessly switched by MPEG-DASH. That is why the configuration is split into multiple groups. When the measurements have been made, all group options are recombined. The settings for the reference video and the parameters have not changed much beyond the additional possibility of selecting multiple settings (see Fig. 1). The order of the encoding parameters is important for a later step in the measurement process. Figure 1 also shows the encoding settings.

The biggest change to the former tool becomes apparent when MPEG-DASH is enabled (see Fig. 2). First, analyzing MPEG-DASH requires multiple changes of the selected format over time, which is difficult to achieve on a short reference signal (e.g. $8 \mathrm{~s}$ ). That is why the reference signal is first looped a selectable number of times to increase its duration. Afterwards, the tool encodes the reference video with different sets of parameters set in the tool (indicated in Fig. 1). With different qualities of encoded videos an additional option is required, which tells the tool how and when to change the selected video format during the measurement. Four options are available for the format selection and the order in which the encoding options are added is important:

- steps (deterministic) - the encoding formats are selected in the sequence in which they were added,

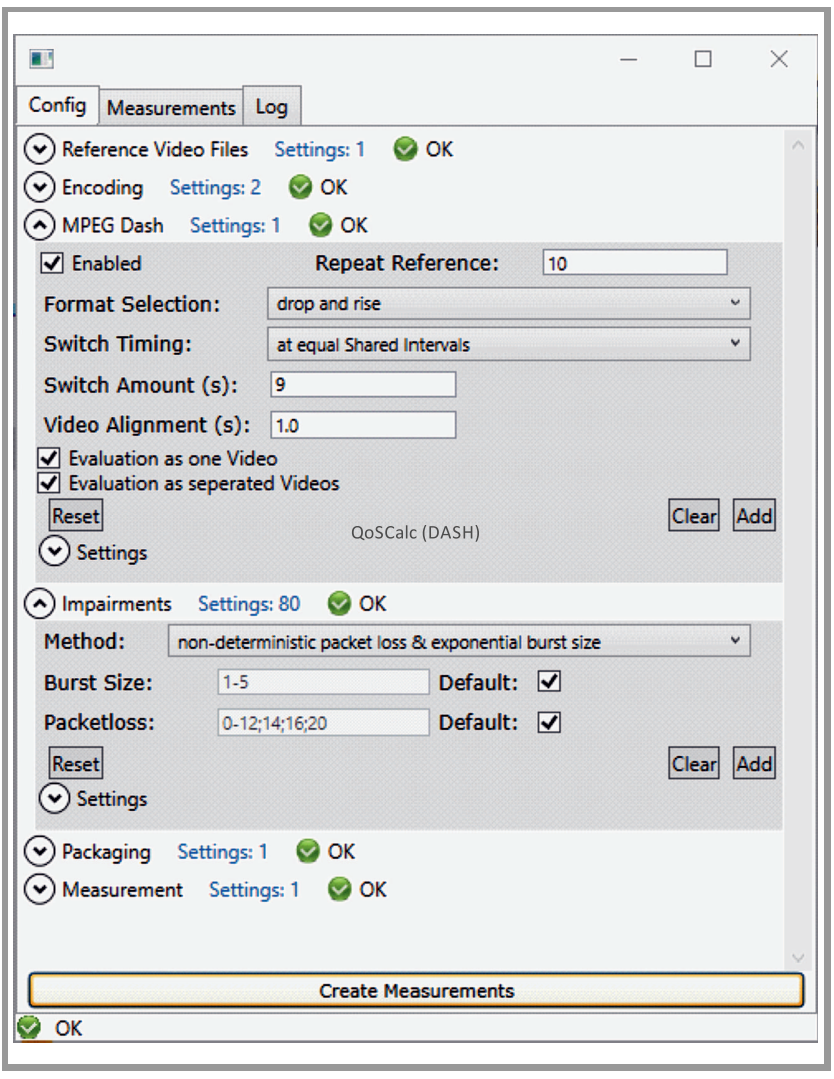

Fig. 2. MPEG-DASH and impairments settings for the QoSCalc(DASH) tool. 
starting from the top again after the last has been reached,

- non-deterministic - the encoding format is selected randomly,

- drop and rise - the encoding formats are selected by using the first setting to the last and then in reverse order,

- rise and drop - opposite to drop and rise by selecting the last to the first and then back down again from the first to the last.

Selecting one of the following three settings determines when the format changes will occur:

- at reference boundary - this will change the format whenever the reference video is repeated (looped),

- at equally spaced intervals - the total length of the video is split in equally sized sequences determined by the number of changes set,

- at non-deterministic intervals - a selected number of changes are distributed randomly over the total time of the measurement (equal distribution).

To accomplish a seamless switch between formats the I-frames have to be available in each format at the same moment in time (frame). FFmpeg [7] as the underlying

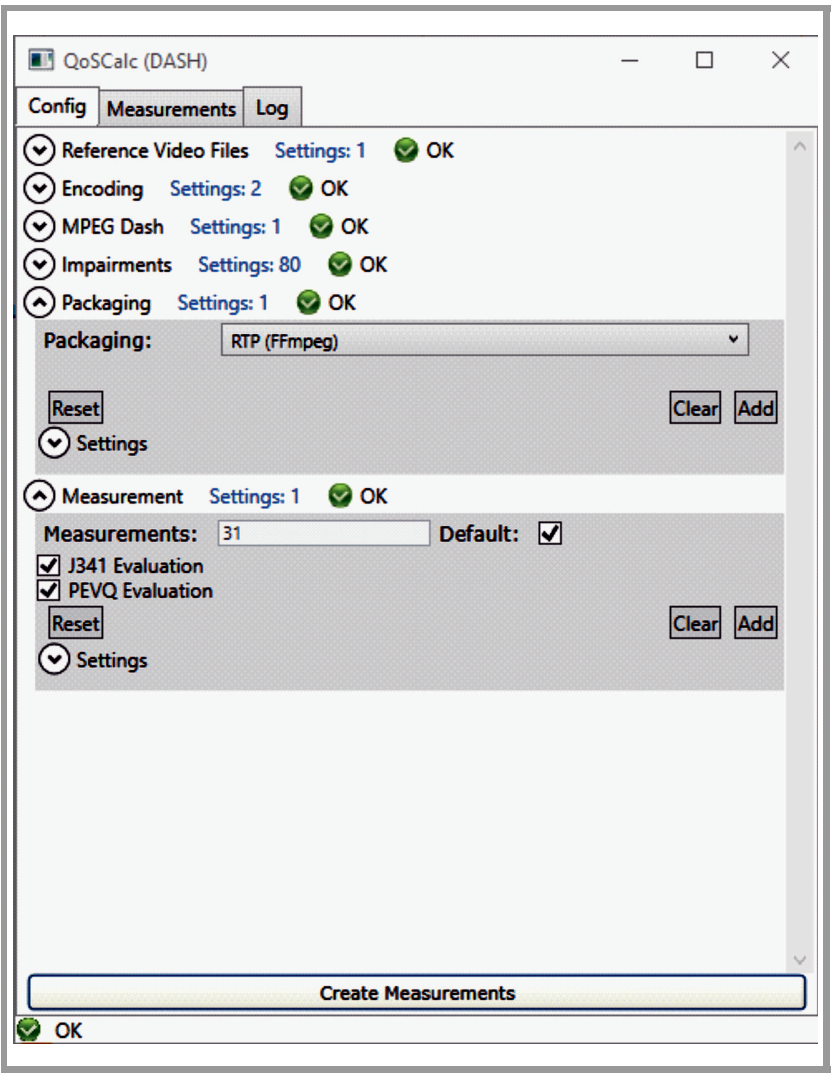

Fig. 3. Packaging and measurement settings for the QoSCalc(DASH) tool. codec library can be told to force an I-frame at constant intervals, programmable in the new configuration window in the video alignment setting. Finally, there is the option in the MPEG-DASH settings with which to select whether the video should be evaluated as one video or each sequence individually or both.

The next group of settings has also changed slightly. Different impairment methods can be selected which determine how packet loss is distributed. The distribution is a function of the selected burst and packet loss values and is applied to each packet of the video stream:

- deterministic packet loss and constant burst size,

- non-deterministic packet loss and constant burst size,

- non-deterministic packet loss and exponential burst size,

- 2-state Markov loss model/BurstR [8].

The packaging options have changed slightly, the three options being MPEG2-TS, RTP and native RTP. The last option provides an additional setting for the selection of the NAL size of each packet. Finally, in the group "measurement" a method can be selected with which to evaluate each measurement and how often each measurement is repeated to gain statistically relevant data (see Fig. 3).

\section{Measurement Scenarios and Measurement Results}

For the first measurements for MPEG-DASH, four slightly different scenarios are analyzed. To make them comparable, some common settings are applied to all scenarios. First, the same reference video from previous papers was used and repeated nine times to give adequately long segments to use five different encoding formats in each scenario in a drop and rise pattern. Drop and rise was selected as it simulates a temporary problem in a video stream in which the quality is first reduced and then increased again. In all scenarios the video was packaged in native RTP with a NAL size of 1500 bytes. An overview of the four scenarios is shown in Table 1.

Table 1

Measurement scenarios

\begin{tabular}{|c|c|c|c|}
\hline Scenario & Codec & Resolution & Bitrate \\
\hline \hline 1 & H.265 & Constant & \\
\cline { 1 - 3 } 2 & H.265 & Variable & \multirow{2}{*}{ Variable } \\
\cline { 1 - 2 } 3 & VP9 & Constant & \\
\hline 4 & VP9 & Variable & \\
\hline
\end{tabular}

Scenarios 2 and 4 use five different resolutions and bitrate combinations which are common in popular web services (see Table 2). 


\section{Scenario 1}

H.265 codec and constant resolution and variable bitrate were used. All encoding parameters except the bitrate remain unchanged for the different formats. The results are shown in Figs. 4 and 5.

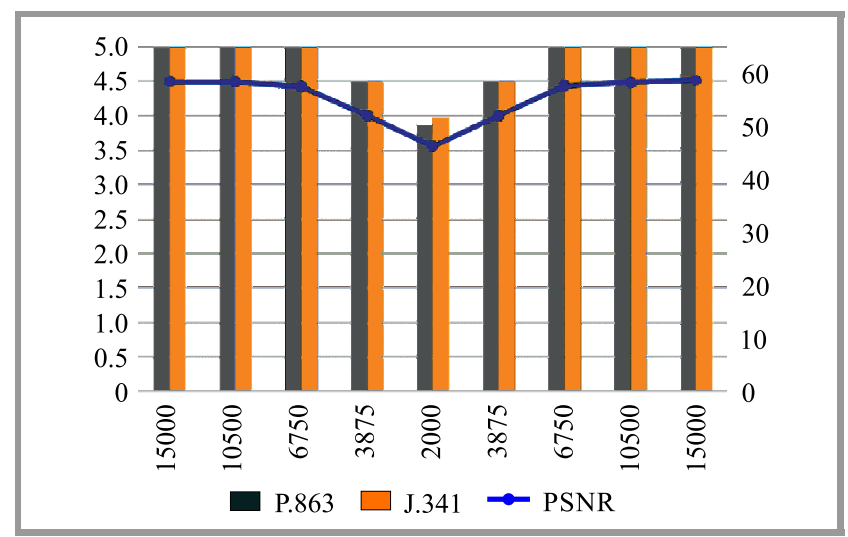

Fig. 4. QoE values as a function of coding rate for Scenario 1 at $0 \%$ packet loss.

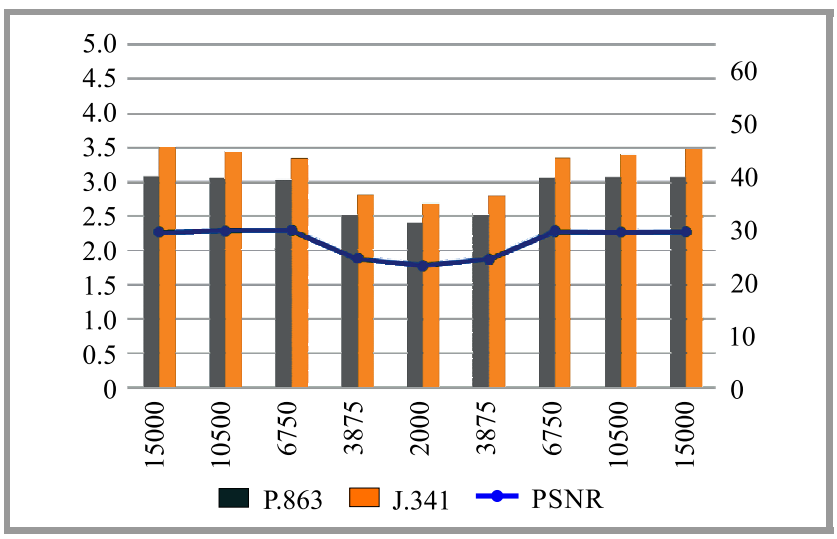

Fig. 5. QoE values as a function of coding rate for Scenario 1 at $1 \%$ packet loss.

As expected, with the decreasing bitrate, the quality of the video service decreases. Packet losses have a large impact on video quality. It is remarkable that even a low packet loss of approximately $1 \%$ has such a great influence on QoE. This behavior is also clearly shown by the PSNR curves. It is evident that there is a strong correlation between QoE values and PSNR values.

\section{Scenario 2}

H.265 codec and variable resolution as well as variable bitrate were used. In this scenario the resolution is changed along with the bitrate. The format details were shown in Table 2. The results are shown in Figs. 6 and 7.

The changes in resolution and bitrate result in a more balanced quality of the video service. Thus, higher quality can be achieved when packet loss occurs by simply changing the bitrate. It is also worth mentioning the strong correlation between QoE values and PSNR values. Additionally, the bandwidth used in Scenario 2 is signifi-
Table 2

Encoding formats for variable resolution scenarios

\begin{tabular}{|c|c|c|}
\hline Format & Resolution [pixels] & Bitrate [kbps] \\
\hline \hline 1 & $1080 \times 1920$ & 4500 \\
\hline 2 & $720 \times 1280$ & 2500 \\
\hline 3 & $480 \times 853$ & 1000 \\
\hline 4 & $360 \times 640$ & 500 \\
\hline 5 & $260 \times 427$ & 300 \\
\hline
\end{tabular}

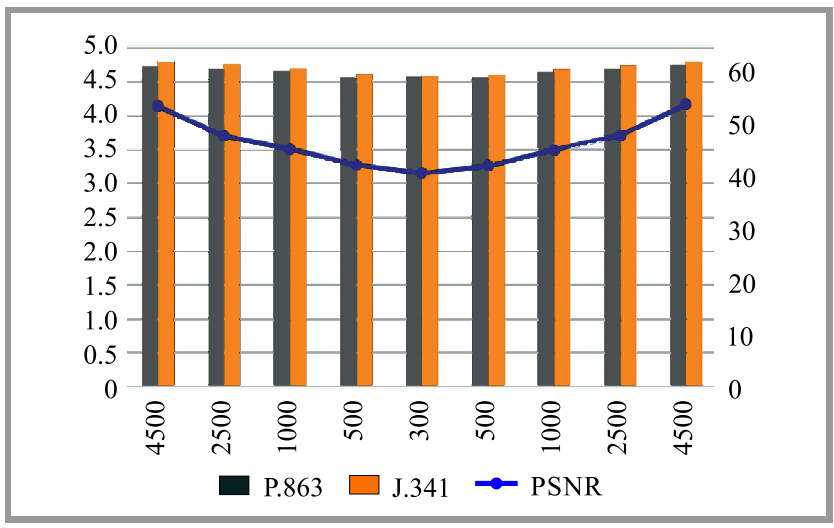

Fig. 6. QoE values as a function of coding rate and resolution for Scenario 2 at $0 \%$ packet loss.

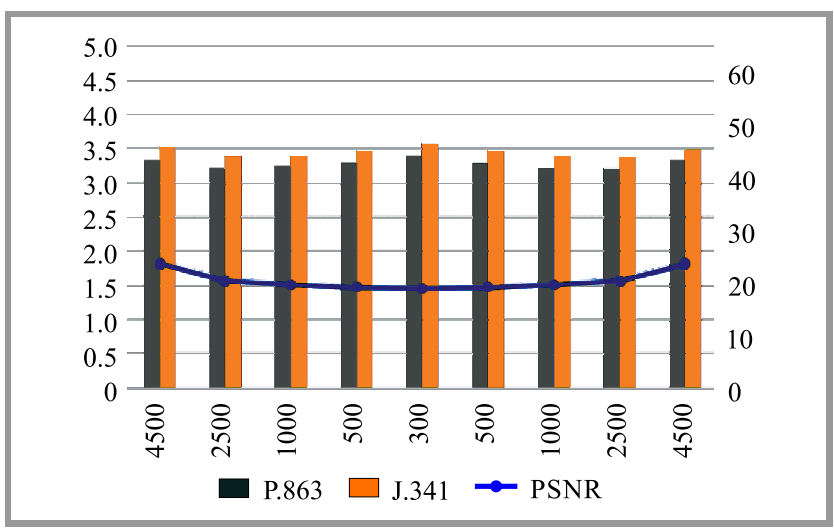

Fig. 7. QoE values versus coding rate and resolution for Scenario 2 at $1 \%$ packet loss.

cantly smaller than that in Scenario 1. This is of great practical benefit, for it reduces the cost of delivering video services.

\section{Scenario 3}

VP9 codec, constant resolution and variable bitrate were used. All encoding parameters except the bitrate remain the same for the different formats.

\section{Scenario 4}

VP9 codec, variable resolution and variable bitrate were used. In this scenario both the resolution and the bitrate were changed.

The results obtained with the VP9 codec in Scenarios 3 and 4 are very similar to the previously discussed results 
using H.265. For this reason and for lack of space they are not presented graphically here. One thing is worth mentioning, however. The benefit of using VP9 is that this codec has a higher compression factor in the encoding process [9]. For this reason, a smaller amount of data needs to be transported. Again, this is of considerable benefit.

\section{Summary and Outlook}

This paper focused on the assessment of the influence of the new MPEG-DASH technique on QoS video streaming over IP. The new technique is flexible and can be implemented to relieve congestion in networks. That can improve the end-to-end QoS. The adaptive bandwidth, that was utilized here, at least ensures image flow even if a deterioration of some of the video sequences is to be expected. That is what makes this technique so strong and is the reason why numerous service providers, such as YouTube, Netflix, Hulu, Twitch, are using it. The analyses conducted during the work described in this paper have shown that it is especially beneficial to use the codec VP9 and to combine the MPEG-DASH technique with changes in image resolution and image coding bitrate.

For the analyses described in this paper the MPEG-DASH technique was used on the RTP transport stream. In practice, however, the technique is most often used in combination with the HTTP protocol. That provides another possibility of transporting video data over networks which further studies will take into consideration. The QoSCalc(DASH) tool will have to be modified first, however, so that it can accommodate the resending of lost video packets. This could be achieved by emulating an HTTP server and an HTTP client in the background of the tool. Further studies in this direction are already being planned.

\section{References}

[1] ISO/IEC DIS 23009-1.2, "Dynamic adaptive streaming over HTTP (DASH)" [Online]. Available: https://www.iso.org/standard/ 57623.html

[2] T. Uhl, "Quality of service everywhere", in Proc. of the 9th Int. Conf. on Adv. Serv. Comput. Service Computation 2017, Athens, Greece, 2017, pp. 28-30.

[3] ITU-T J.247 (08/2008), "Objective perceptual multimedia video quality measurement in the presence of a full reference" [Online]. Available: http://www.itu.int/rec/T-REC-J.247-200808-I/ 2017.11.03

[4] ITU-T J.341 (03/2016), “Objective perceptual multimedia video quality measurement of HDTV for digital cable television in the presence of a full reference" [Online]. Available: http://www.itu.int/rec/T-RECJ.341-201101-I/en/2017.11.03

[5] "Dynamic Adaptive Streaming over HTTP" [Online]. Available: https://en.wikipedia.org/wiki/Dynamic_Adaptive_Streaming_over_ HTTP/2017.11.03

[6] T. Uhl and H. Jürgensen, "New tool for examining QoS in the IPTV service", in Proc. of the World Telecom. Congr. WTC'14, Berlin, Germany, 2014.
[7] "FFmpeg (current Window builds)" [Online]. Available: http://FFmpeg.zeranoe.com/builds/2017.11.03

[8] S. Paulsen, "QoS/QoE-Modelle für den Dienst Voice over IP (VoIP)", Dissertation, University Hamburg, Department of Information Technology, 2016 (in German).

[9] M. Řeřábek and T. Ebrahimi, "Comparison of compression efficiency between HEVC/H.265 and VP9 based on subjective assessments" [Online]. Available: https://infoscience.epfl.ch/record/200925/files/ article-vp9-submited-v2.pdf/2017.11.03

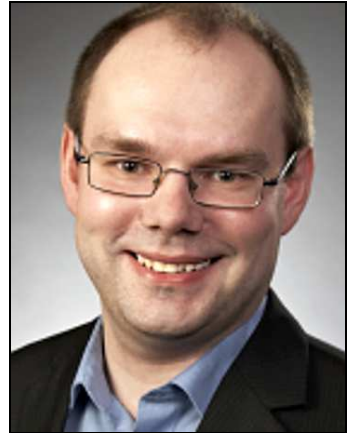

Christian Hoppe received his B.Eng. in Communications Technology from the Flensburg University of Applied Sciences, Germany, in 2010 and his M.Sc. in Information Technology from the Kiel University of Applied Science, Germany, in 2016. Today, he works as a Software Engineer for Nextragen Solutions GmbH in Flensburg, Germany. His main activities cover the following areas: quality assurance for Triple Play services and medical imaging solutions.

E-mail: christian.hoppe@nextragen-solutions.de Nextragen Solutions GmbH Flensburg

Heideland 1

D-24976 Handewitt, Germany

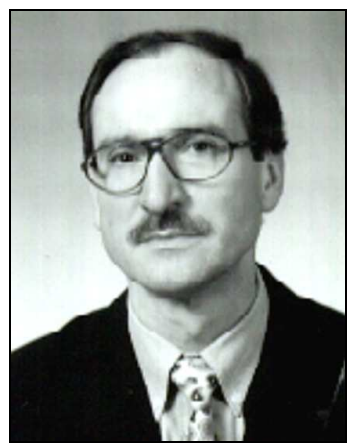

Tadeus Uhl received his M.Sc. in Telecommunications from the Academy of Technology and Agriculture in Bydgoszcz, Poland in 1975, his Ph.D. from Gdańsk University of Technology, Gdańsk, Poland in 1982 and his D.Sc. from University at Dortmund, Germany in 1990. Since 1992 he has worked as Professor at the Institute of Communications Technology, Flensburg University of Applied Sciences, Germany and, in addition, since 2013, as Professor at the Institute of Transport Engineering and Economics, Maritime University of Szczecin, Poland. His main activities cover the following areas: traffic engineering, performance analysis of communications systems, measurement and evaluation of communication protocols, QoS and QoE by Triple Play services, Ethernet and IP technology. He is an author or co-author of five books and 130 papers on the subjects of LAN, WAN and NGN.

E-mail: t.uhl@am.szczecin.pl Maritime University of Szczecin

Henryka Pobożnego 11

70-507 Szczecin, Poland 